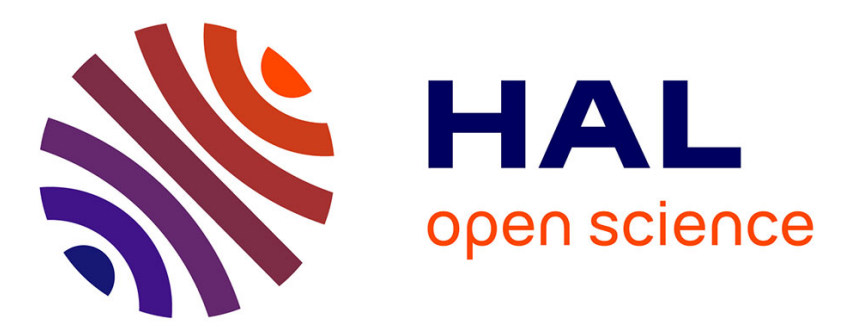

\title{
Alternative formulation to incorporate forcing terms in a lattice Boltzmann scheme with central moments
}

\author{
Alessandro de Rosis
}

\section{To cite this version:}

Alessandro de Rosis. Alternative formulation to incorporate forcing terms in a lattice Boltzmann scheme with central moments. Physical Review E , 2017, 95 (2), pp.023311. 10.1103/PhysRevE.95.023311 . hal-01724822

\section{HAL Id: hal-01724822 \\ https://hal.science/hal-01724822}

Submitted on 7 Mar 2018

HAL is a multi-disciplinary open access archive for the deposit and dissemination of scientific research documents, whether they are published or not. The documents may come from teaching and research institutions in France or abroad, or from public or private research centers.
L'archive ouverte pluridisciplinaire HAL, est destinée au dépôt et à la diffusion de documents scientifiques de niveau recherche, publiés ou non, émanant des établissements d'enseignement et de recherche français ou étrangers, des laboratoires publics ou privés. 


\title{
Alternative formulation to incorporate forcing terms in a lattice Boltzmann scheme with central moments
}

\author{
Alessandro De Rosis* \\ Department of Biomedical Engineering Technion-Israel Institute of Technology, 32000 Haifa, Israel \\ (Received 7 December 2016; revised manuscript received 19 January 2017; published 23 February 2017)

\begin{abstract}
Within the framework of the central-moment-based lattice Boltzmann method, we propose a strategy to account for external forces in two and three dimensions. Its numerical properties are evaluated against consolidated benchmark problems, highlighting very high accuracy and optimal convergence. Moreover, our derivations are
\end{abstract} \\ light and intelligible.
}

DOI: 10.1103/PhysRevE.95.023311

\section{INTRODUCTION}

Originally derived to remove the statistical noise affecting the lattice gas automata [1,2], during the last 20 years the lattice Boltzmann method (LBM) has undergone a vast series of developments and improvements. Nowadays, it is a robust and established technique to perform numerical simulations of viscous fluids [3-10]. One of the keys to its success lies in the simplicity of the governing equation, a first-order partial differential one called the lattice Boltzmann equation (LBE). It consists of the well-known "collide-and-stream" process to compute the evolution of the particle distribution functions (or populations), such that its averaged behavior recovers the dynamics of fluid motion. While the streaming stage is a simple shift of data along discrete characteristic particle directions, the collision process is the most physically salient and delicate aspect of the method.

The most popular collision operator is the BhatnagarGross-Krook (BGK) one [11], which forces the populations to relax to attractors, known as local equilibria, derived as a second-order-truncated Taylor expansion in the Mach number. It is also known as the single-relaxation-time LBM, as all the populations relax at a common rate. It has been proved that the BGK LBM is consistent with the solution of the Navier-Stokes equations for incompressible flow with a second order of accuracy [12]. Later, d'Humières [13] proposed to decompose the collision stage in a space of moments. Each moment can relax with a proper rate, hence the notation multiple relaxation time (MRT), and the postcollision populations are reconstructed from the collided moments. If compared to the BGK operator, the adoption of the MRT has proved to increase the stability of the LB scheme due to the removal of spurious modes [14,15].

Recently, a novel collision kernel has been proposed by Geier et al. [16]. A new family of moments has been introduced, which relax in a frame moving with the fluid. The members of this class are called "central" moments (CMs), in contrast to the "raw" moments of the MRT, which are defined in a frame at rest $[13,17]$. As for the MRT, central moments are relaxed independently. In a CM-based kernel, one of the crucial points to construct the collision operator is

\footnotetext{
*Also at Université Lyon, Ecole Centrale de Lyon, CNRS, Laboratoire de Mécanique des Fluides et d'Acoustique, F-69134 Ecully Cedex, France; alessandro@bm.technion.ac.il
}

represented by the binomial theorem, allowing us to relate raw and central moments. Specifically, the postcollision value of a CM at a certain order depends on the lower-order raw ones, and not vice versa. This particular one-way coupling implies that the collision is configured as a structured hierarchical pyramidal sequence of relaxation in an ascending order of moments, hence the name "cascaded" LBM. These models are characterized by very high properties in terms of accuracy, stability, and convergence [18-25].

An important aspect of any fluid flow simulation is the possibility to account for the presence of external forces (e.g., gravity or Coriolis forces). Within the LB community, Guo et al. [26] developed a very valuable approach that has been widely adopted. Another interesting model is represented by the exact difference method (EDM) [27], which has been successfully adopted in Ref. [21] to investigate multiphase flows by means of CMs. More recently, Premnath et al. [28] have presented a methodology to incorporate forcing terms in the cascaded LBM. Again, the binomial theorem has been adopted to construct such a forcing operator. Despite its effectiveness and excellent numerical properties, it may lead to cumbersome practical implementations that are particularly evident in three dimensions [29,30].

This paper proposes a different strategy to model external forces in a CM-based collision kernel. Starting from the definition provided in Ref. [31], we build a forcing term according to the nonorthogonal basis of moments. Differently from Refs. [28,29], here the binomial theorem is not required, as the usage of raw moments is avoided, and the formulation results in an intelligible derivation. The model is validated against very-well-defined test cases, demonstrating its excellent numerical performance.

\section{PRESENT APPROACH}

In this section, our approach is presented. It is worth noting that the present work focuses only on forcing terms. Therefore, a detailed discussion about central-moment-based collision kernels is not reported herein.

\section{A. Two-dimensional model}

The two-dimensional discrete LB equation predicts the evolution in space, $\boldsymbol{x}=[x, y]$, and time $t$ of the particle distribution functions $\left|f_{i}\right\rangle=\left[f_{0}, f_{1}, f_{2}, f_{3}, f_{4}, f_{5}, f_{6}, f_{7}, f_{8}\right]^{\top}$, where |\rangle and $T$ denote a column vector and the transpose 
operator, respectively. Populations move on a fixed Cartesian square lattice along the generic link $i=0 \ldots 8$ with velocity $\mathbf{c}_{i}=\left[\left|c_{x i}\right\rangle,\left|c_{y i}\right\rangle\right]$ defined according to the two-dimensional nine-velocity lattice (D2Q9) model [6],

$$
\begin{aligned}
& \left|c_{x i}\right\rangle=[0,1,0,-1,0,1,-1,-1,1]^{\top}, \\
& \left|c_{y i}\right\rangle=[0,0,1,0,-1,1,1,-1,-1]^{\top} .
\end{aligned}
$$

Let us introduce the external source term $\left|\mathcal{F}_{i}\right\rangle=$ $\left[\mathcal{F}_{0}, \mathcal{F}_{1}, \mathcal{F}_{2}, \mathcal{F}_{3}, \mathcal{F}_{4}, \mathcal{F}_{5}, \mathcal{F}_{6}, \mathcal{F}_{7}, \mathcal{F}_{8}\right]^{\top}$. Then, the BGK LBE reads as follows,

$$
\begin{gathered}
f_{i}\left(\boldsymbol{x}+\Delta t \mathbf{c}_{i}, t+\Delta t\right)=f_{i}(\boldsymbol{x}, t)+\Omega(\boldsymbol{x}, t) \\
+\frac{1}{2}\left[\mathcal{F}_{i}(\boldsymbol{x}, t)+\mathcal{F}_{i}\left(\boldsymbol{x}+\Delta t \mathbf{c}_{i}, t+\Delta t\right)\right],
\end{gathered}
$$

where the time step is $\Delta t=1$ and $\Omega(\boldsymbol{x}, t)$ is the collision operator. In order to remove the implicitness in Eq. (1), the following transformation [28,29] is applied,

$$
f_{i}^{\dagger}(\boldsymbol{x}, t)=f_{i}(\boldsymbol{x}, t)-\frac{1}{2} \mathcal{F}_{i}(\boldsymbol{x}, t) .
$$

Then, the LBE equation can be rewritten as

$$
f_{i}^{\dagger}\left(\boldsymbol{x}+\Delta t \mathbf{c}_{i}, t+\Delta t\right)=f_{i}^{\dagger}(\boldsymbol{x}, t)+\Omega(\boldsymbol{x}, t)+\mathcal{F}_{i}(\boldsymbol{x}, t) .
$$

As usual, this process is split into two parts, which are called collision,

$$
f_{i}^{\dagger \star}(\boldsymbol{x}, t)=f_{i}^{\dagger}(\boldsymbol{x}, t)+\Omega(\boldsymbol{x}, t)+\mathcal{F}_{i}(\boldsymbol{x}, t),
$$

and streaming,

$$
f_{i}^{\dagger}\left(\boldsymbol{x}+\Delta t \mathbf{c}_{i}, t+\Delta t\right)=f_{i}^{\dagger \star}(\boldsymbol{x}, t),
$$

respectively. Here and henceforth, the superscript $\star$ denotes postcollision quantities and the dependence on $\boldsymbol{x}$ and $t$ is implicitly assumed. Macroscopic variables can be computed as

$$
\rho=\sum_{i} f_{i}^{\dagger}, \quad \rho \boldsymbol{u}=\sum_{i} f_{i}^{\dagger} \mathbf{c}_{i}+\frac{1}{2} \boldsymbol{F} \Delta t,
$$

where $\rho$ is the fluid density, and $\boldsymbol{u}=\left[u_{x}, u_{y}\right]$ and $\boldsymbol{F}=\left[F_{x}, F_{y}\right]$ are the flow velocity and external force vectors, respectively.

Now, let us build the forcing operator in terms of central moments. According to He et al. [31], the forcing term can be written as

$$
\mathcal{F}_{i}=\frac{\boldsymbol{F}}{\rho} \cdot \frac{\overline{\mathbf{c}}_{i}}{c_{s}^{2}} f_{i}^{\mathrm{eq}},
$$

where the equilibrium populations are

$$
f_{i}^{\text {eq }}=w_{i} \rho\left[1+\frac{\mathbf{c}_{i} \cdot \boldsymbol{u}}{c_{s}^{2}}+\frac{\left(\mathbf{c}_{i} \cdot \boldsymbol{u}\right)^{2}}{2 c_{s}^{4}}-\frac{\boldsymbol{u} \cdot \boldsymbol{u}}{2 c_{s}^{2}}\right] .
$$

Lattice directions shifted by the local fluid velocity are

$$
\overline{\mathbf{c}}_{i}=\left[\left|\bar{c}_{x i}\right\rangle,\left|\bar{c}_{y i}\right\rangle\right]
$$

where

$$
\begin{aligned}
\left|\bar{c}_{x i}\right\rangle & =\left|c_{x i}-u_{x}\right\rangle, \\
\left|\bar{c}_{y i}\right\rangle & =\left|c_{y i}-u_{y}\right\rangle .
\end{aligned}
$$

The lattice sound speed is $c_{s}=1 / \sqrt{3}$ and the weighting factors are $w_{0}=4 / 9, w_{1 \ldots 4}=1 / 9$, and $w_{5 \ldots 8}=1 / 36$. We adopt a basis defined as

$$
\overline{\mathcal{T}}=\left[\left|\bar{T}_{0}\right\rangle, \ldots,\left|\bar{T}_{i}\right\rangle, \ldots,\left|\bar{T}_{8}\right\rangle\right] .
$$

The reader can refer to Refs. [21,28,32] for the expressions of each component of the basis. Consistently, CMs of the forcing term are defined as

$$
\left|\xi_{i}\right\rangle=\left[\xi_{0}, \ldots, \xi_{8}\right]^{\top} .
$$

We propose to compute these quantities as

$$
\left|\xi_{i}\right\rangle=\overline{\mathcal{T}}^{\top}\left|\mathcal{F}_{i}\right\rangle
$$

resulting in the following expressions:

$$
\begin{aligned}
\xi_{0}= & 0, \\
\xi_{1}= & F_{x}, \\
\xi_{2}= & F_{y}, \\
\xi_{3}= & -3\left(F_{x} u_{x}+F_{y} u_{y}\right)\left(u_{x}^{2}+u_{y}^{2}\right), \\
\xi_{4}= & -3\left(F_{x} u_{x}+F_{y} u_{y}\right)\left(u_{x}^{2}-u_{y}^{2}\right), \\
\xi_{5}= & 3 u_{x} u_{y}\left(F_{x} u_{x}+F_{y} u_{y}\right), \\
\xi_{6}= & 9 F_{x} u_{x}^{3} u_{y}+9 F_{y} u_{x}^{2} u_{y}^{2}+F_{y} / 3, \\
\xi_{7}= & 9 F_{x} u_{x}^{2} u_{y}^{2}+9 F_{y} u_{x} u_{y}^{3}+F_{x} / 3, \\
\xi_{8}= & -18 u_{x}^{2} u_{y}^{2}\left(F_{x} u_{x}+F_{y} u_{y}\right) \\
& -3 u_{x} u_{y}\left(F_{x} u_{y}+F_{y} u_{x}\right)-F_{x} u_{x}^{3}-F_{y} u_{y}^{3} .
\end{aligned}
$$

It is worth stressing that the practical implementation of the set of $\left|\xi_{i}\right\rangle=\left[\xi_{0}, \ldots, \xi_{8}\right]^{\top}$ represents an easy task. In fact, one can simply solve Eq. (14), instead of coding directly the above-mentioned formulas. This is particularly worthy to be noted if a three-dimensional model is adopted. Let us denote as $\left|k_{i}\right\rangle=\left[k_{0}, \ldots, k_{8}\right]^{\top},\left|k_{i}^{\mathrm{eq}}\right\rangle=\left[k_{0}^{\mathrm{eq}}, \ldots, k_{8}^{\mathrm{eq}}\right]^{\top}$, and $\left|k_{i}^{\star}\right\rangle=\left[k_{0}^{\star}, \ldots, k_{8}^{\star}\right]^{\top}$ the basis of precollision, equilibrium, and postcollision central moments of the populations. The members of the first and second groups are evaluated according to Ref. [32]. Then, the collision stage is performed as

$$
k_{i}^{\star}=k_{i}+\omega_{i}\left(k_{i}^{\mathrm{eq}}-k_{i}\right)-\frac{1}{2} \xi_{i}, \quad i=1 \ldots 8,
$$

where $\omega_{i}$ is the relaxation frequency associated with the moment $k_{i}$. Notice that $k_{0}$ is collision invariant. The presence of the last term in the right-hand side obeys Eq. (3). Furthermore, postcollision populations $\left|f_{i}^{\dagger \star}\right\rangle=\left[f_{0}^{\dagger \star}, \ldots, f_{8}^{\dagger \star}\right]^{\top}$ are reconstructed by solving $\overline{\mathcal{T}}^{\top}\left|f_{i}^{\dagger \star}\right\rangle=\left|k_{i}^{\star}\right\rangle$ and, eventually, these are streamed by Eq. (6) [32].

In the Supplemental Material, a script [33] allows the reader to perform the symbolic manipulations to obtain all the involved quantities.

\section{B. Three-dimensional model}

In three dimensions, let us consider the D3Q27 model. In this case, the Eulerian basis is $\boldsymbol{x}=[x, y, z]$, and the fluid velocity and external force vectors are $\boldsymbol{u}=\left[u_{x}, u_{y}, u_{z}\right]$ and $\boldsymbol{F}=\left[F_{x}, F_{y}, F_{z}\right]$, respectively. The transformed populations are defined as $\left|f_{i}^{\dagger}\right\rangle=\left[f_{0}^{\dagger}, \ldots, f_{26}^{\dagger}\right]^{\top}$ and the lattice velocity 
vectors are

$$
\begin{aligned}
\left|c_{x i}\right\rangle= & {[0,1,-1,0,0,0,0,1,-1,1,-1,1,-1,1,-1,} \\
& 0,0,0,0,1,-1,1,-1,1,-1,1,-1]^{\top}, \\
\left|c_{y i}\right\rangle= & {[0,0,0,1,-1,0,0,1,1,-1,-1,0,0,0,0,1,} \\
& -1,1,-1,1,1,-1,-1,1,1,-1,-1]^{\top}, \\
\left|c_{z i}\right\rangle= & {[0,0,0,0,0,1,-1,0,0,0,0,1,1,1,-1,} \\
& 1,1,-1,-1,1,1,1,1,-1,-1,-1,-1]^{\top} .
\end{aligned}
$$

Moreover, the weights are set to $w_{0}=8 / 27, w_{1 \ldots 6}=2 / 27$, $w_{7 \ldots 18}=1 / 54, w_{19 \ldots 26}=1 / 216$. Equations (11) are completed by

$$
\left|\bar{c}_{z i}\right\rangle=\left|c_{z i}-u_{z}\right\rangle
$$

as now $\overline{\mathbf{c}}_{i}=\left[\left|\bar{c}_{x i}\right\rangle,\left|\bar{c}_{y i}\right\rangle,\left|\bar{c}_{z i}\right\rangle\right]$. Now, the integer index $i$ assumes the values $i=0 \ldots 26$. Let us employ the following basis,

$$
\overline{\mathcal{T}}=\left[\left|\bar{T}_{0}\right\rangle, \ldots,\left|\bar{T}_{i}\right\rangle, \ldots,\left|\bar{T}_{26}\right\rangle\right],
$$

whose components are outlined in Refs. [29,30]. In this case, the central moments are $\left|\xi_{i}\right\rangle=\left[\xi_{0}, \ldots, \xi_{26}\right]^{\top}$ and can be easily computed, again, by Eq. (14). Postcollision central moments are evaluated by Eq. (16). Then, populations are reconstructed and streamed, as usual. The resultant expressions of $\xi_{0 . .26}$ are quite long and are not reported herein. However, as for the twodimensional case, a script [33] is added to the Supplemental Material to derive the entire three-dimensional formulation. Here, we have derived the scheme for the D2Q9 and D3Q27 models. Notice that the same procedure can be adopted for any lattice velocity space.

\section{NUMERICAL EXPERIMENTS}

Here, we report our results concerning three different tests. In each scenario, the density field is initialized as $\rho(\boldsymbol{x}, 0)=\rho_{0}$ everywhere, with $\rho_{0}=1$, and the D2Q9 model is adopted. Moreover, the performance of the present model, $\mathcal{S}_{\text {Present }}$, is compared to the one achieved by implementing the exact difference method [27], $\mathcal{S}_{\mathrm{EDM}}$, and the model proposed by Premnath et al. [28], $\mathcal{S}_{\text {Premnath }}$.

\section{A. Driving forces: Four-rolls mill}

Let us consider a square periodic box of size $N \times N$, where the fluid is initially at rest. Let us apply a constant force field, that is,

$$
\boldsymbol{F}(\boldsymbol{x})=\phi[\sin (x) \sin (y), \cos (x) \cos (y)] .
$$

with $\phi=2 v u_{0} \psi^{2}$. Then, the pressure and velocity fields must converge to a steady state that reads as follows,

$$
\begin{aligned}
& p(\boldsymbol{x})=p_{0}\left[1-\frac{u_{0}^{2}}{4 c_{s}^{2}}[\cos (2 \psi x)-\cos (2 \psi y)]\right], \\
& \boldsymbol{u}(\boldsymbol{x})=u_{0}[\sin (\psi x) \sin (\psi y), \cos (\psi x) \cos (\psi y)],
\end{aligned}
$$

where $u_{0}=10^{-3}, \psi=2 \pi / N$, and $p_{0}=\rho_{0} c_{s}^{2}$. This test is known as four-rolls mill and is a modification of the canonical Taylor-Green vortex [34]. Simulations characterized by different grid sizes are carried out, i.e., $N=[8,16,32,64,128]$, at a

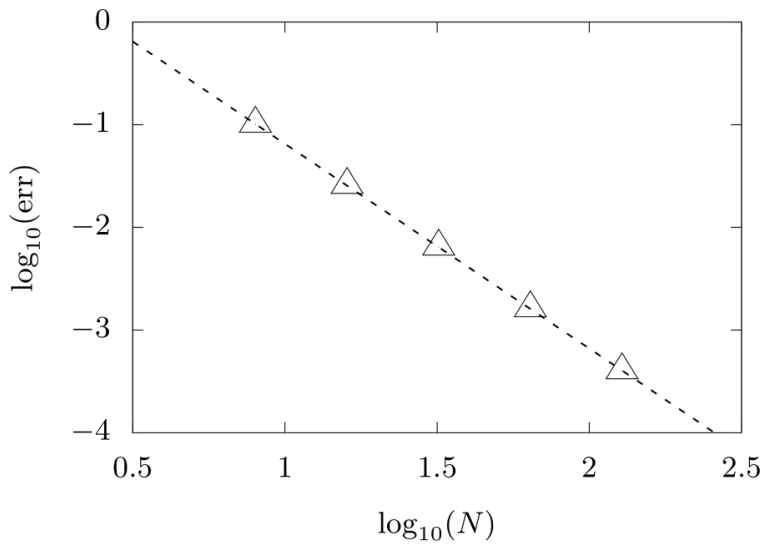

FIG. 1. Four-rolls mill: The slope of the line fitting our results (triangles) indicates a convergence rate of 1.997 .

Reynolds number $\operatorname{Re}=u_{0} N / v$ equal to 100 . The performance of our scheme is elucidated by computing the relative discrepancy between analytical predictions and numerical findings. For this purpose, the vectors $\mathbf{r}_{\mathrm{an}}$ and $\mathbf{r}_{\text {num }}$ are introduced, storing the values of the velocity field from Eq. (21) and those provided by our numerical experiments, respectively. Then, the relative error is computed as

$$
\text { err }=\frac{\left\|\mathbf{r}_{\text {an }}-\mathbf{r}_{\text {num }}\right\|}{\left\|\mathbf{r}_{\text {an }}\right\|}
$$

and is depicted in Fig. 1 as a function of the grid dimension. An excellent convergence rate equal to 1.997 is found that is totally consistent with the second-order nature of the LB equation. We repeated the experiment by adopting the EDM and the scheme developed in Ref. [28]. In Table I, the relative error achieved by each method is reported for every grid resolution. The model in Ref. [28] manifests an outlier for the coarsest grid resolution. It is found that the EDM possesses the best performance, even if a very slight mismatch emerges with respect to findings achieved by the remaining models. In fact, each one shows a convergence rate $\sim 2$.

\section{B. Magnetohydrodynamic forces: Orszag-Tang vortex}

By considering an electrically conductive fluid, at the macroscopic level the fluid dynamics obeys the incompressible Navier-Stokes equations accounting for the Lorentz force, completed by the magnetic induction equation for the spacetime evolution of the magnetic field, namely, $\boldsymbol{b}=\left[b_{x}, b_{y}, b_{z}\right]$.

TABLE I. Four-rolls mill: Percentage relative errors and convergence rate achieved by different forcing schemes. Convergence rates (CR) are computed by excluding findings at $N=8$.

\begin{tabular}{lccc}
\hline \hline$N$ & $\mathcal{S}_{\text {Present }}$ & $\mathcal{S}_{\text {EDM }}$ & $\mathcal{S}_{\text {Premnath }}$ \\
\hline 8 & 10.120 & 10.112 & 28.487 \\
16 & 2.5608 & 2.5567 & 2.558 \\
32 & 0.64223 & 0.64013 & 0.6407 \\
64 & 0.16129 & 0.16023 & 0.16042 \\
128 & 0.04023 & 0.03969 & 0.03984 \\
$\mathrm{CR}$ & 1.997 & 2.003 & 2.001 \\
\hline \hline
\end{tabular}


The problem is solved through the two-population model proposed in Ref. [35]. Let us also introduce the current vector $\boldsymbol{j}=\left[j_{x}, j_{y}, j_{z}\right]$. Consistently, it is possible to define the Lorentz force as $\boldsymbol{F}_{L}=\boldsymbol{j} \times \boldsymbol{b}$. In Ref. [35], it has been argued that the effect of the Lorentz force in the Navier-Stokes equations can be accounted for directly in the equilibrium distribution functions of the velocity field. Conversely, here we propose a different approach. Specifically, we use $\boldsymbol{F}_{L}=\left[F_{L x}, F_{L y}, F_{L z}\right]$ as an external force that is inserted in a CM-based algorithm by means of our proposed scheme. To obtain the Lorentz force, the current field is required. Its components can be evaluated locally from the particle distribution functions [36] as

$$
j_{\alpha}=-\frac{\omega_{m}}{\theta}\left[\sum_{i}\left(c_{i \beta} g_{i \gamma}-c_{i \gamma} g_{i \beta}\right)-2\left(u_{\beta} b_{\gamma}-u_{\gamma} b_{\beta}\right)\right],
$$

where $\alpha, \beta$, and $\gamma$ span the Eulerian basis. Notice that the quantity $\theta$ is equal to $c_{s}^{2}$ in two dimensions, whereas it assumes the values of $1 / 4$ in a three-dimensional model $[35,36]$. The relaxation frequency $\omega_{m}$ is linked to the magnetic resistivity $\eta$ as $\eta=\left(\frac{1}{\omega_{m}}-\frac{1}{2}\right) \theta$.

It can be observed that the fluid velocity $\boldsymbol{u}$ depends on $\boldsymbol{F}_{L}$ that is in turn related to $\boldsymbol{u}$ itself. In order to make accurate computations, we develop an iterative scheme to address the correct value of the Lorentz force at each grid point. Within the typical time step and at the generic location $\boldsymbol{x}$, this process can be summarized as follows.

(1) Compute the magnetic field, the density, and Lorentz force-free velocity $\tilde{\boldsymbol{u}}$. The latter is

$$
\tilde{\boldsymbol{u}}=\frac{1}{\rho} \sum_{i} f_{i} \mathbf{c}_{i}
$$

(2) Define the quantity $j_{x}^{\ddagger}, j_{y}^{\ddagger}, j_{z}^{\ddagger}$ as

$$
\begin{aligned}
& j_{x}^{\ddagger}=\sum_{i}\left(c_{i y} g_{i z}-c_{i z} g_{i y}\right), \\
& j_{y}^{\ddagger}=\sum_{i}\left(c_{i z} g_{i x}-c_{i x} g_{i z}\right), \\
& j_{z}^{\ddagger}=\sum_{i}\left(c_{i x} g_{i y}-c_{i y} g_{i x}\right),
\end{aligned}
$$

(3) Estimate the tentative value of the current:

$$
\begin{aligned}
& j_{x}^{(0)}=-\frac{\omega_{m}}{\theta}\left[j_{x}^{\ddagger}-2\left(\tilde{u}_{y} b_{z}-\tilde{u}_{z} b_{y}\right)\right], \\
& j_{y}^{(0)}=-\frac{\omega_{m}}{\theta}\left[j_{y}^{\ddagger}-2\left(\tilde{u}_{z} b_{x}-\tilde{u}_{x} b_{z}\right)\right], \\
& j_{z}^{(0)}=-\frac{\omega_{m}}{\theta}\left[j_{z}^{\ddagger}-2\left(\tilde{u}_{x} b_{y}-\tilde{u}_{y} b_{x}\right)\right] .
\end{aligned}
$$

(4) Perform the following procedure starting with the iteration counter $p=0$.

(a) Evaluate the three components of the Lorentz force:

$$
\begin{aligned}
& F_{L x}^{(p)}=b_{z} j_{y}^{(p)}-b_{y} j_{z}^{(p)}, \\
& F_{L y}^{(p)}=b_{x} j_{z}^{(p)}-b_{z} j_{x}^{(p)}, \\
& F_{L z}^{(p)}=b_{y} j_{x}^{(p)}-b_{x} j_{y}^{(p)} .
\end{aligned}
$$

(b) Correct the fluid velocity:

$$
\boldsymbol{u}^{(p)}=\tilde{\boldsymbol{u}}+\frac{1}{2 \rho} \boldsymbol{F}_{L}^{(p)} .
$$

(c) Update the current:

$$
\begin{aligned}
& j_{x}^{(p+1)}=-\frac{\omega_{m}}{\theta}\left[j_{x}^{\ddagger}-2\left(u_{y}^{(p)} b_{z}-u_{z}^{(p)} b_{y}\right)\right], \\
& j_{y}^{(p+1)}=-\frac{\omega_{m}}{\theta}\left[j_{y}^{\ddagger}-2\left(u_{z}^{(p)} b_{x}-u_{x}^{(p)} b_{z}\right)\right], \\
& j_{z}^{(p+1)}=-\frac{\omega_{m}}{\theta}\left[j_{z}^{\ddagger}-2\left(u_{x}^{(p)} b_{y}-u_{y}^{(p)} b_{x}\right)\right] .
\end{aligned}
$$

(d) Check the convergence criterion:

$$
\frac{\left|j^{(p+1)}-j^{(p)}\right|}{\left|j^{(p+1)}\right|} \leqslant 10^{-4}
$$

with $j=\|\boldsymbol{j}\|$.

(e) If Eq. (30) is not satisfied, go to step 4(a) and increase the counter $p$ by 1 . Otherwise, exit.

Then, the final value of $\boldsymbol{F}_{L}$ is incorporated in the LBE. Interestingly, the above-sketched algorithm is drastically simplified in two dimensions, where the current vector possesses only one nonzero component.

Let us assume a two-dimensional configuration. We test our formulation against the Orszag-Tang vortex problem [35,37]. A square periodic box of length $2 \pi$ is considered with a Reynolds number of $\operatorname{Re} \approx 628$. Initial conditions are defined as

$$
\begin{gathered}
\boldsymbol{u}(\boldsymbol{x}, 0)=v_{0}[-\sin (h y), \sin (h x)], \\
\boldsymbol{b}(\boldsymbol{x}, 0)=b_{0}[-\sin (h y), \sin (2 h x)],
\end{gathered}
$$

where $h=2 \pi / M$, with $M=512$ being the number of grid points in the $x$ and $y$ directions. The values of $v_{0}$ and $b_{0}$ are chosen in order to carry out numerical experiments at a Mach number $\mathrm{Ma}=v_{0} / c_{s} \approx 0.0028$. Notice that the Reynolds and magnetic Prandtl numbers are defined as $\operatorname{Re}=v_{0} N / v$ and $\operatorname{Pr}=v / \eta=1$, respectively. In Table II, our findings are compared to results from pseudospectral simulations [35] in terms of peak values of the current, $j_{\max }=\max _{x} j(x)$, and vorticity, $\zeta_{\max }=\max _{x}|\nabla \times \boldsymbol{u}(\boldsymbol{x})|$, at two representative time instants, i.e., $t=0.5,1$. Let us introduce the quantities err 1, err $_{2}$, and $\operatorname{err}_{3}$. These are computed through Eq. (22), hence measuring the accuracy of the results achieved by the present model, the EDM, and the scheme in Ref. [28] against the reference values [35]. Our values are highly satisfactory. In fact, the relative difference between the present and spectral results from Ref. [35] exhibit a slight discrepancy ranging between $0.1 \%$ and $0.7 \%$. Interestingly, all the adopted schemes are accurate, with our proposed one showing the best performance for all the monitoredbrk quantities. The contour maps of the magnetic, current, velocity, and vorticity fields are depicted in Fig. 2. It is possible to appreciate that the adopted grid resolution is able to capture fine flow features. Moreover, progressively larger gradients of all the reported quantities appear in time. This observation corroborates findings in Table II, where the errors are found to increase with $t$. In addition, it is consistent with the seminal contribution in Ref. [35], where the same time-dependent pattern of the 
TABLE II. Orszag-Tang vortex: Findings from our scheme, EDM [27], model in Ref. [28], and reference spectral values from Ref. [35] in terms of the peak value of the current, $j_{\max }$, and vorticity, $\zeta_{\max }$, at two representative time instants, together with the percentage relative errors.

\begin{tabular}{|c|c|c|c|c|c|c|c|c|}
\hline & $t$ & Ref. [35] & $\mathcal{S}_{\text {Present }}$ & $\mathcal{S}_{\mathrm{EDM}}$ & $\mathcal{S}_{\text {Premnath }}$ & $\operatorname{err}_{1}(\%)$ & $\operatorname{err}_{2}(\%)$ & $\operatorname{err}_{3}(\%)$ \\
\hline \multirow[t]{2}{*}{$j_{\max }$} & 0.5 & 18.24 & 18.26 & 18.27 & 18.26 & 0.1096 & 0.1645 & 0.1096 \\
\hline & 1 & 46.59 & 46.66 & 46.69 & 46.66 & 0.1502 & 0.2146 & 0.1502 \\
\hline \multirow[t]{2}{*}{$\zeta_{\max }$} & 0.5 & 6.758 & 6.756 & 6.756 & 6.755 & 0.0296 & 0.0296 & 0.0444 \\
\hline & 1 & 14.20 & 14.11 & 14.10 & 14.10 & 0.6338 & 0.7042 & 0.7042 \\
\hline
\end{tabular}
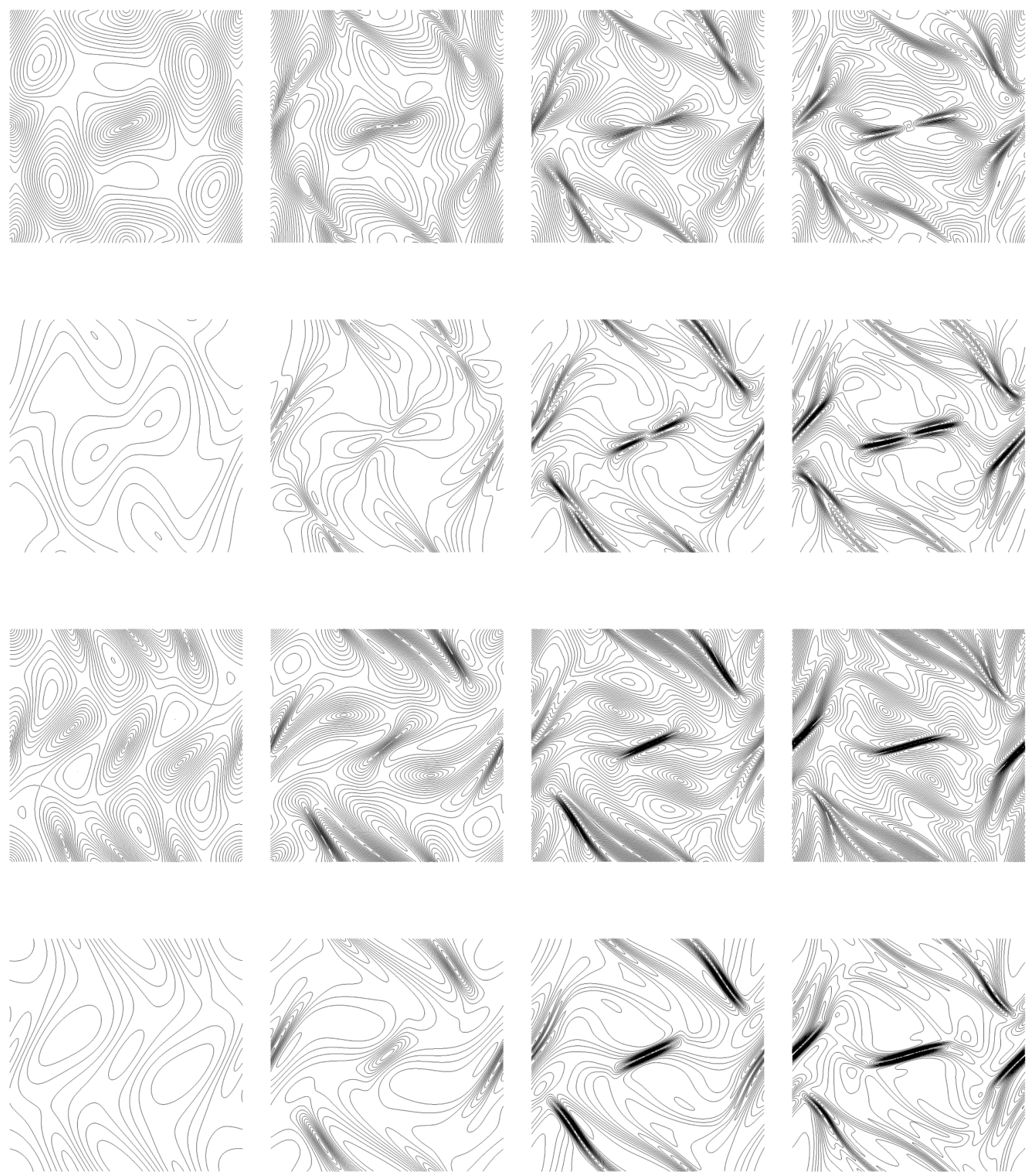

FIG. 2. Orszag-Tang vortex: Contour maps of the magnitude of velocity (first row), vorticity (second row), magnetic field (third row), and current (fourth row) at $t=0.25$ (first column), 0.5 (second column), 0.75 (third column), and 1 (fourth column). Numbering proceeds from the top to the bottom of the page and from left to right. 
TABLE III. Oscillating cylinder in a quiescent fluid: The quantities $e_{1}$ and $e_{2}$ are provided by different approaches and reference values from the literature $[39,44,45]$.

\begin{tabular}{ccccccc}
\hline \hline & Ref. [39] & Ref. [44] & Ref. [45] & $\mathcal{S}_{\text {Present }}$ & $\mathcal{S}_{\text {EDM }}$ & $\mathcal{S}_{\text {Premnath }}$ \\
\hline$e_{1}$ & 2.09 & 2.10 & 2.10 & 2.10 & 2.10 & 2.10 \\
$e_{2}$ & 1.45 & 1.45 & 1.45 & 1.45 & 1.45 & 1.45 \\
\hline \hline
\end{tabular}

error is experienced. Finally (and again in agreement with Ref. [35]), notice that the presence of finite values of resistivity and viscosity visibly affects our plots, especially if compared to the perfectly conducting and inviscid case in Ref. [38].

\section{Forces prescribing the no-slip condition on an immersed moving boundary: Oscillating cylinder in a quiescent fluid}

The last comparison is performed by investigating the flow physics induced by the harmonic motion of a cylinder of diameter $D=100$ in a calm viscous fluid [39]. The presence of a moving body is accounted for by the immersed boundary method [40]. In short, it generates a body force that enforces the no-slip condition at the fluid-boundary interface. Therefore, we use this quantity in our proposed forcing operator. The interested reader can refer to Ref. [41] for an in-depth discussion about the adopted lattice Boltzmann-immersed boundary model. The fluid domain consists of $55 D \times 35 D$ lattice points in the horizontal and vertical directions, respectively. The cylinder is initially placed in the center of the fluid domain and it undergoes a horizontal harmonic motion of period $T$ and velocity $v(t)=-V \cos (2 \pi t / T)$, with $V=0.01$ being the peak value. The governing parameters of the flow are the Reynolds number, $\operatorname{Re}=V D / v=100$, and the KeuleganCarpenter number, $\mathrm{KC}=V T / D=5$.

According to Ref. [42], the horizontal force acting on the cylinder, namely, $Q_{x}$, can be computed by a semiempirical formula, i.e.,

$$
Q_{x}=-\frac{1}{2} e_{1} \rho D v|v|-\frac{1}{4} e_{2} \pi \rho D^{2} \dot{v}
$$
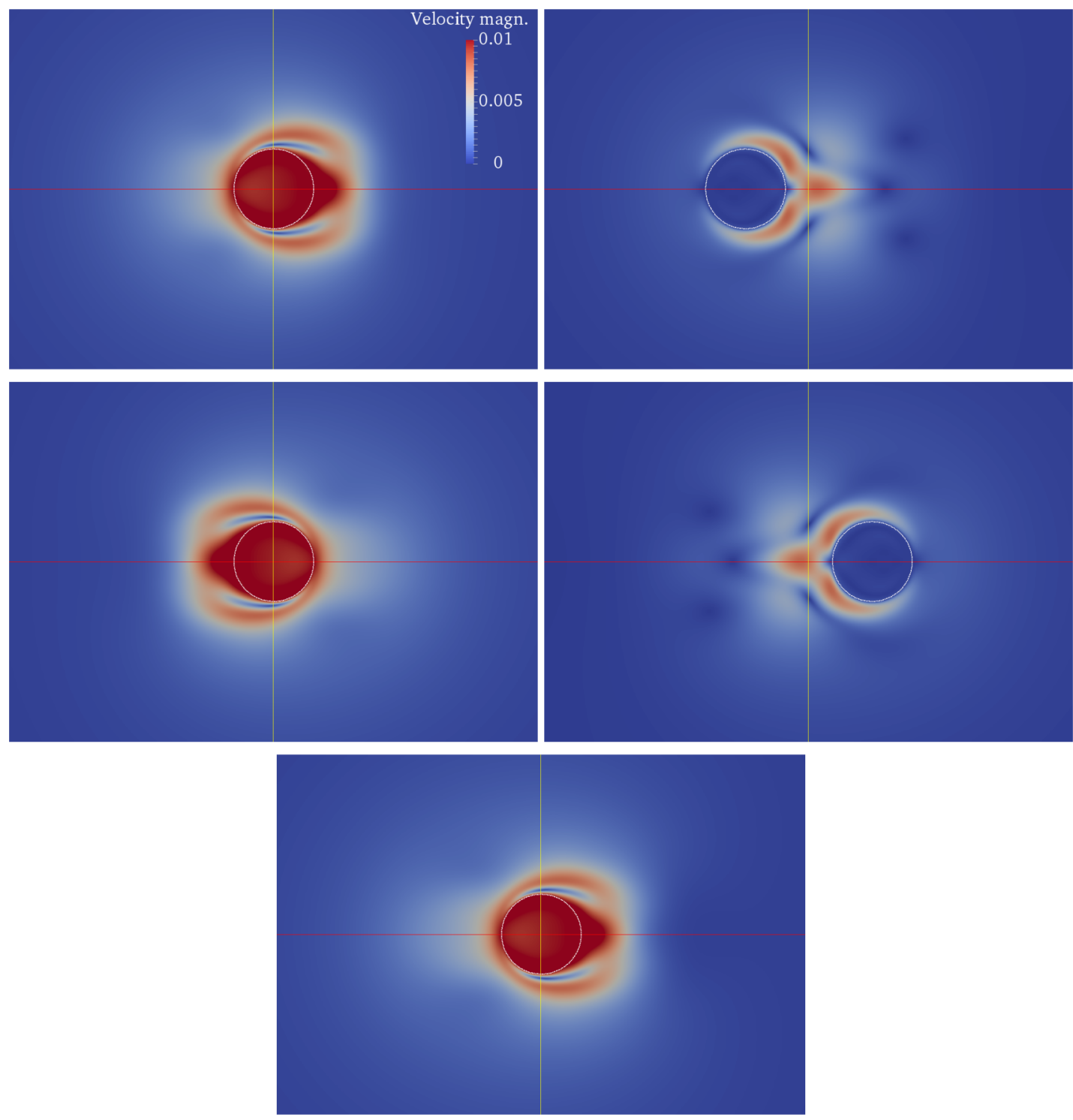

FIG. 3. Oscillating cylinder in a quiescent fluid: Map of velocity magnitude at different time instants, i.e., $t / T=2$ (top left), 2.25 (top right), 2.5 (middle left), 2.75 (middle right), and 3 (bottom). 
where a superimposed dot indicates the time derivative. The quantities $e_{1}$ and $e_{2}$ are the drag and added-mass coefficients [42]. These are evaluated by a non-linear least-square fit with the MATLAB curve fitting toolbox [43]. Specifically, the force signal has been fitted by neglecting the first cycle of oscillation, thus avoiding any issue related to initial transient dynamics. Then, two subsequent cycles have been used. Our findings and those provided by implementing the schemes in Refs. [27,28] are reported in Table III and are compared to reference values from the literature $[39,44,45]$. An excellent agreement is shown.

Finally, the velocity field induced by the motion of the cylinder is shown in Fig. 3 during a period of oscillation, i.e., $t / T \in[2: 3]$. At $t / T=2.25$ and $t / T=2.75$, the cylinder stops its motion. Here, it is particularly worth noting that the fluid travels around its surface and the velocity vanishes close to the boundary. This manifests clearly that the impenetrability of the cylinder surface is very well enforced by our scheme.

\section{CONCLUSIONS}

Under the same assumption of the works by Premnath et al. [28,29] (i.e., the representation of the forcing term according to He et al. [31]), we have demonstrated that there is an alternative way to construct an external source operator. In particular, the most advantageous aspects of our proposed approach are as follows.

(1) Generality: The method can be developed for any lattice space without ad hoc derivations.
(2) Intelligible derivations: The analytical formulation is light, even in three dimensions, as it does not require the binomial theorem and the usage of raw moments.

(3) Practical implementation: No cumbersome expressions are involved in the computations, as CMs of the forcing term can be computed by Eq. (14).

Moreover, the model has shown very high accuracy and convergence properties that are fully consistent with other existing approaches.

\section{ACKNOWLEDGMENTS}

This work was done in the framework of the "Programme Avenir Lyon Saint-Etienne" of the Université de Lyon (ANR-11-IDEX-0007), within the Program "Investissements d'Avenir" operated by the French National Research Agency. The research leading to these results has received funding from the People Programme (Marie Curie Actions) of the European Union's Seventh Framework Programme (FP7/2007-2013) under REA Grant Agreement No. PCOFUND-GA-2013-609102, through the PRESTIGE programme coordinated by Campus France. This article is based upon work from COST Action MP1305, supported by COST (European Cooperation in Science and Technology). The author is grateful to Professor E. Lévêque for insightful discussions about the central-momentbased scheme, and to Professor W. J. T. Bos and Robert Chahine for useful hints about magnetohydrodynamic forces. The author would like to thank the anonymous referees, who have improved the quality of this work with their comments.
[1] G. R. McNamara and G. Zanetti, Phys. Rev. Lett. 61, 2332 (1988).

[2] D. A. Wolf-Gladrow, Lattice-Gas Cellular Automata and Lattice Boltzmann Models: An Introduction (Springer, Berlin, 2004).

[3] F. Higuera, S. Succi, and R. Benzi, Europhys. Lett. 9, 345 (1989).

[4] R. Benzi, S. Succi, and M. Vergassola, Phys. Rep. 222, 145 (1992).

[5] S. Chen and G. Doolen, Annu. Rev. Fluid Mech. 30, 329 (1998).

[6] S. Succi, The Lattice Boltzmann Equation for Fluid Dynamics and Beyond (Clarendon, Oxford, UK, 2001).

[7] H. Chen, S. Kandasamy, S. Orszag, R. Shock, S. Succi, and V. Yakhot, Science 301, 633 (2003).

[8] S. Succi, Europhys. Lett. 109, 50001 (2015).

[9] S. Succi, Philos. Trans. R. Soc., A 374, 20160151 (2016).

[10] A. De Rosis, E. Lévêque, S. Ubertini, and S. Succi, Europhys. Lett. 113, 18001 (2016).

[11] P. Bhatnagar, E. Gross, and M. Krook, Phys. Rev. 94, 511 (1954).

[12] H. Chen, S. Chen, and W. Matthaeus, Phys. Rev. A 45, R5339 (1992).

[13] D. d'Humières, Philos. Trans. R. Soc., A 360, 437 (2002).

[14] H. Chen, C. Teixeira, and K. Molvig, Int. J. Mod. Phys. C 08, 675 (1997).

[15] J. Latt and B. Chopard, Math. Comput. Simul. 72, 165 (2006).

[16] M. Geier, A. Greiner, and J. G. Korvink, Phys. Rev. E 73, 066705 (2006).

[17] P. Asinari, Phys. Rev. E 78, 016701 (2008).

[18] M. Geier, A. Greiner, and J. Korvink, Int. J. Mod. Phys. C 18, 455 (2007).
[19] M. Geier, Int. J. Numer. Methods Fluids 56, 1249 (2008).

[20] M. Geier, A. Greiner, and J. Korvink, Eur. Phys. J.: Spec. Top. 171, 55 (2009).

[21] D. Lycett-Brown and K. Luo, Comput. Math. Appl. 67, 350 (2014).

[22] Y. Ning, K. N. Premnath, and D. V. Patil, Int. J. Numer. Methods Fluids 82, 59 (2016).

[23] M. Geier, M. Schönherr, A. Pasquali, and M. Krafczyk, Comput. Math. Appl. 70, 507 (2015).

[24] M. Geier, A. Fakhari, and T. Lee, Phys. Rev. E 91, 063309 (2015).

[25] A. De Rosis and E. Lévêque, Comput. Math. Appl. 72, 1616 (2016).

[26] Z. Guo, C. Zheng, and B. Shi, Phys. Rev. E 65, 046308 (2002).

[27] A. Kupershtokh, in Proceedings of the 5th International EHD Workshop (University of Poitiers, Poitiers, France, 2004), pp. 241-246.

[28] K. N. Premnath and S. Banerjee, Phys. Rev. E 80, 036702 (2009).

[29] K. Premnath and S. Banerjee, J. Stat. Phys. 143, 747 (2011).

[30] A. De Rosis, Phys. Rev. E 95, 013310 (2017).

[31] X. He, S. Chen, and G. D. Doolen, J. Comput. Phys. 146, 282 (1998).

[32] A. De Rosis, Europhys. Lett. 116, 44003 (2017).

[33] See Supplemental Material at http://link.aps.org/supplemental/ 10.1103/PhysRevE.95.023311 for performing all the computations to obtain $k_{i}, k_{i}^{\mathrm{eq}}$, and $f_{i}^{\star}$, and to derive the entire three-dimensional formulation. 
[34] G. Taylor and A. Green, Proc. R. Soc. London, Ser. A 158, 499 (1937).

[35] P. J. Dellar, J. Comput. Phys. 179, 95 (2002).

[36] M. Pattison, K. Premnath, N. Morley, and M. Abdou, Fusion Eng. Des. 83, 557 (2008).

[37] S. A. Orszag and C.-M. Tang, J. Fluid Mech. 90, 129 (1979).

[38] R. Grauer and C. Marliani, Phys. Plasmas 5, 2544 (1998).

[39] H. Dütsch, F. Durst, S. Becker, and H. Lienhart, J. Fluid Mech. 360, 249 (1998).

[40] C. Peskin, Acta Numerica 11, 479 (2002).
[41] A. De Rosis, S. Ubertini, and F. Ubertini, J. Sci. Comput. 61, 477 (2014).

[42] J. Morison, J. Johnson, and S. Schaaf, J. Petrol. Technol. 2, 149 (1950).

[43] MATLAB, version 9.0 (R2016a) (The MathWorks Inc., Natick, MA, 2016).

[44] B. Uzunoğlu, M. Tan, and W. Price, Int. J. Numer. Methods Eng. 50, 2317 (2001).

[45] R. Yuan, C. Zhong, and H. Zhang, J. Comput. Phys. 296, 184 (2015). 